\title{
DESIGN OF A DUAL KEYBOARD
}

\author{
${ }^{1}$ Ragavi, V. and ${ }^{2}$ G. Geetha \\ ${ }^{1}$ Assistant Professor, Department of Computer Applications, Sathyabama University, Chennai, India \\ ${ }^{2}$ Professor and Associate Dean, School of Computer Science and Applications, Lovely Professional University, Punjab, India
}

Received 2013-08-29, Revised 2013-09-19; Accepted 2013-10-02

\begin{abstract}
The design of a computer keyboard with dual function is proposed. This computer keyboard called Dual Keyboard can function both as a normal keyboard and as a pressure sensitive keyboard. The proposed device has a switch that decides the function. The keyboard makes use of sensors placed beneath the keys to measure the pressure applied on the key by the user. This device has many applications. In this study, it is applied to mitigate Denial of Service (DoS) attack.
\end{abstract}

Keywords: Pressure Sensitive, Computer, Sensor, Security, FSR, DoS

\section{INTRODUCTION}

Keyboard is the standard input device. A small modification to the traditional computer keyboard is attempted to make the computer keyboard more innovative and interactive.

The proposed computer keyboard has dual functionality. It works as a normal keyboard for regular works. For special applications, it works as a pressure sensitive keyboard, since pressure sensitivity is not always required.

The basic idea for pressure sensitive keyboard was drawn from Electronic Musical keyboards that have switches under each key. Depressing a key connects a circuit, which triggers tone generation. This motivated the research, to place pressure sensors under each key to measure the pressure of the depressed key. The Dual Keyboard while working as a pressure sensitive keyboard is used to mitigate Denial of Service, which is discussed in Section 4.

\subsection{Literature Review}

There is a considerable amount of work done in pressure sensitivity for many interactive systems. Buxton (2007) outlined touch computing systems and pressure sensitive devices. Ramos et al. (2004) explored the design space of using the continuous pressure sensing Corresponding Author: Ragavi, V., Assistant Professor, Department of Computer Application, Sathyabama University, Chennai, India capabilities of styluses to operate multi-state widgets. The authors had also presented the taxonomy of widgets. Many studies were conducted in using pressure sensitivity to enhance GUI objects.

Ramos and Balakrishnan (2005; 2007) introduced pressure marks-pen strokes, where the variations in pressure made it possible to indicate both a selection and an action simultaneously. Zagler et al. (2013) in their FASTY program have examined methods to improve text generation for disabled people. This program included the use of pressure sensitive keyboard with an interface box. Loy et al. (2005) have investigated how keystroke pressure strengthened the security over traditional password-based authentication system. They compared its performance with that of the conventional timing based keystroke technique. The authors also investigated the use of combined keystroke pressure and latency for the verification process. Hidetoshi and Kurihara (2007) proposed an authentication system using keystroke dynamics. In the study the authors illustrated how sensed pressure can be used for accuracy measurement and durability against intrusion using keylogger. Bergadano et al. (2002) in their article, addressed the problem of user authentication via keystroke dynamics. They presented a new keystroke analysis technique that helped to solve two of the problems related to keystroke analysis: The mentioned intrinsic 
variability of typing and the possibility of typing errors. Dietz et al. (2009) discussed about a practical pressure sensitive keyboard. They replaced the existing keyboard membranes. Henderson et al. (2002) used signals resulting from tapping a rhythm on a pressure sensor and explored this waveform for authentication. The Piezo-electric and Piezo resistive sensors are screen printed on a thin layer, which is then glued with an object. The sensors are robust, thin (70 microns) and inexpensive. Henderson and Hartel (2000) have stated that the sensors can easily be integrated into objects of various shapes, including smart cards and handheld devices such as PDAs and tools.

\section{PROPOSED SYSTEM}

A Computer keyboard with a dual mechanism is proposed. An On/Off switch is used which determines the function of the keyboard. When the switch is "On", the keyboard functions as a pressure sensitive keyboard. When the switch is "Off" the keyboard functions as a normal keyboard. Figure 1 Shows the flowchart of the dual keyboard functioning.

In the process of designing the pressure sensitivity in keyboard, various experiments were conducted. Section 3.1 explains the experiment, observation and inference for the selection of sensors. Section 3.2 describes the experiment, observation and inference to decide the placement of sensor. The system configuration incorporating the results of section 3.1 and 3.2 is presented in Section 3.3. The test results and output is shown in Section 3.4 .

\section{Experiment 1}

\subsection{Selection of Sensors}

The selection of the sensor material was done after a considerable study. The sensors considered were: P-876 Patch transducer, Piezo capacitor and FSR. The comparison of the above said materials was carried out on four dimensions i.e., the size of the sensor, the cost of each sensor, availability and accuracy in output. The comparison is shown in Table $\mathbf{1 .}$

P-876-Piezo sensitive sensors are cost significant. The analog circuitry to monitor the sensor is very complex. Adhesive of the sensors to the metal covers requires a special adhesive.

Piezo Capacitor - The sensors do not provide steady state information on the press of the sensor.

The smaller the size of the piezo sensors, the cost is high as compared to FSR.

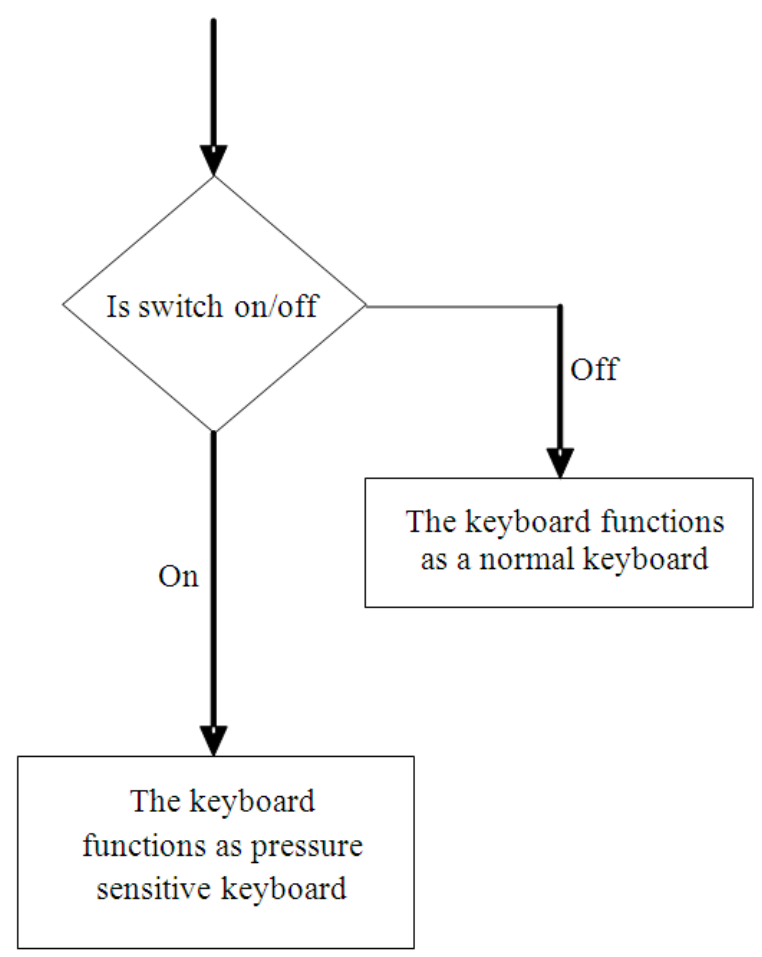

Fig. 1. Flow chart for dual keyboard functioning

The force applied on the keyboard by normal human lies between 0-20 lbs. The sensors considered were tested. Table 2 shows the reading of the corresponding voltage when finger force is applied on the sensors. From the table we can infer that FSR was the only sensor to respond to the above said human applied pressure. The other two sensors needed very high pressure than usual. Figure $\mathbf{2}$ shows the comparison chart.

From the above Table 1 and 2, Fig. 2 and from the above discussion, it is concluded that FSR is the best choice.

The shape of FSR is circular, with $12 \mathrm{~mm}$ in diameter and $0.55 \mathrm{~mm}$ in thickness. The sensor consists of two thin, flexible polyester sheets which have electrically conductive electrodes deposited in varying patterns. The inside surface of one sheet forms a row pattern while the inner surface of the other employs a column pattern. The intersection of these rows and columns creates a sensing cell. The spacing between the rows and columns varies according to sensor application and can be as small as $\sim 0.5 \mathrm{~mm}$. 
Table 1. Comparison of pressure sensors

\begin{tabular}{llllll}
\hline Sensors & Shape & Size & Cost & Availability & Accuracy \\
\hline P-876 & Square & $10 * 10 \mathrm{~mm}$ & High & Under development & $\pm 3 \%$ \\
FSR & Circle & $12 \mathrm{~mm}$ & Low & Easily available & $\pm 3 \%$ \\
Piezo capacitor & Rectangle & $6 * 3.7 \mathrm{~mm}$ & Very high & Easily available & $\pm 2 \%$ \\
\hline
\end{tabular}

Table 2. Comparison of pressure sensors with respect to force applied

\begin{tabular}{lllll}
\hline Force $(\mathrm{lb})$ & Force $(\mathrm{N})$ & $\begin{array}{l}\text { FSR-voltage } \\
\text { across R }\end{array}$ & $\begin{array}{l}\text { P-876 voltage } \\
\text { across R }\end{array}$ & $\begin{array}{l}\text { Piezo capacitor } \\
\text { voltage across R }\end{array}$ \\
\hline None & None & $0 \mathrm{~V}$ & $0 \mathrm{~V}$ & $0 \mathrm{~V}$ \\
$0.04 \mathrm{lb}$ & $0.2 \mathrm{~N}$ & $1.3 \mathrm{~V}$ & $0 \mathrm{~V}$ & $0 \mathrm{~V}$ \\
$0.22 \mathrm{lb}$ & $1 \mathrm{~N}$ & $3.1 \mathrm{~V}$ & $0.1 \mathrm{~V}$ & $0 \mathrm{~V}$ \\
$2.2 \mathrm{lb}$ & $10 \mathrm{~N}$ & $4.5 \mathrm{~V}$ & $1.2 \mathrm{~V}$ & $0 \mathrm{~V}$ \\
$22 \mathrm{lb}$ & $100 \mathrm{~N}$ & $4.9 \mathrm{~V}$ & $2.5 \mathrm{~V}$ & $100 \mathrm{mamp}$ \\
\hline
\end{tabular}

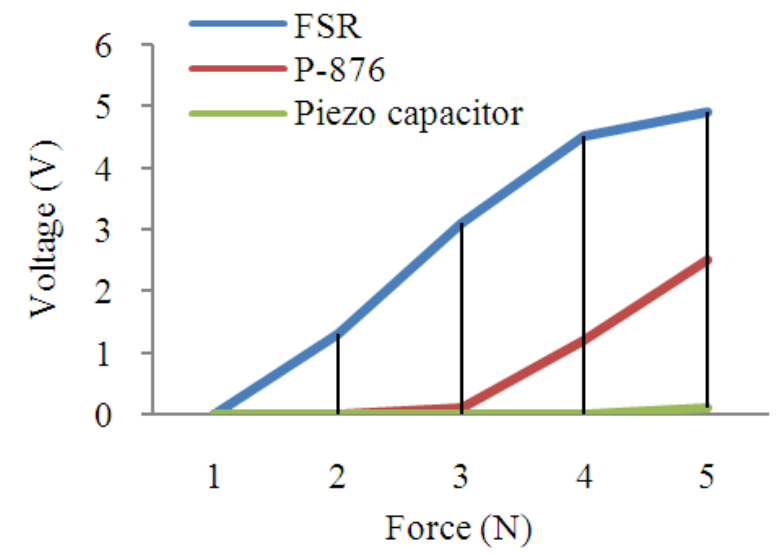

Fig. 2. Comparison chart

\section{Experiment 2}

\subsection{Placement of Sensors Inside the Keyboard}

In the traditional computer keyboard, there is a rubber dome beneath the key, below the rubber dome there is a Printed Circuit Board (PCB) which forms the top layer or top contacts, the next comes the space layer with a hole in it. Following the space layer is the bottom layer or bottom contacts which is again another printed circuit board. Usually when a key is pressed the dome touches the top contact which squeezes the space layer and touches the bottom contacts, the circuit is thus closed and the signal is passed. The traditional keyboard layers are shown in the Fig. 3.

In the design of Computer Keyboard by Hidetoshi and Kurihara (2007) have not mentioned anything about the placement of sensors. But they have used cork as a sensor attachment.

In the design of Pressure sensitive Keyboard by Dietz et al. (2009) they have replaced the top contact, spacer layer and bottom contacts with specially designed membrane.

Initially the sensors were placed between the key and the dome in a traditional keyboard. After prolonged usage, it was found that the sensors did not long last and got damaged.

The sensors cannot be placed elsewhere after the dome because the circuit has to close in order to register the key stroke.

So, the sensor was placed below the bottom contacts. The placement of Sensor beneath the bottom contacts is shown in Fig 4.

When the key is pressed, the dome strikes the top contact which squeezes the space layer and touches the bottom layer, the force applied on the key is sensed by the pressure sensor which is placed under Neath the bottom layer. The horizontal circuit is closed separately when the space layer touches the bottom layer, thus allowing current to run through it. This indicates the "Key Down" or else it is "Key Up".

The results of these experiments were incorporated in the final design.

\subsection{System Configuration}

Figure 5 Shows the system configuration of the Dual Keyboard. The design tells how the system is functioning. The sensor placed keyboard (Dual Keyboard) is configured with the PC. The data from each sensor are sent to the ATMega Microcontroller through a Multiplexer and an A/D converter. It is converted to 10-bit digital data in $1.0000 \mathrm{kHz}$ rate. An 8-bit data is retained temporarily in a buffer. The size of the buffer is 1,048,576-bit, so that it can preserve the measured data $65,536 \mathrm{~ms}$. Depending on the PC's request the unsent data from the buffer is sent to the PC via USB. The rate of transmission of data is $12 \mathrm{Mbps}$. Each request is issued to PC by hitting the key. The data is sent through the USB. 


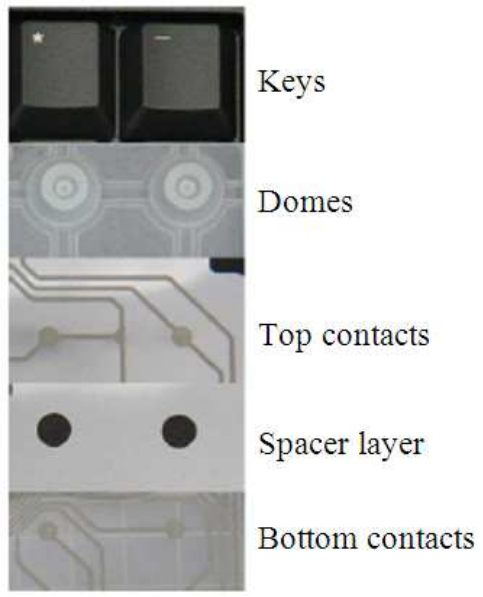

Fig. 3. Traditional membrane keyboard

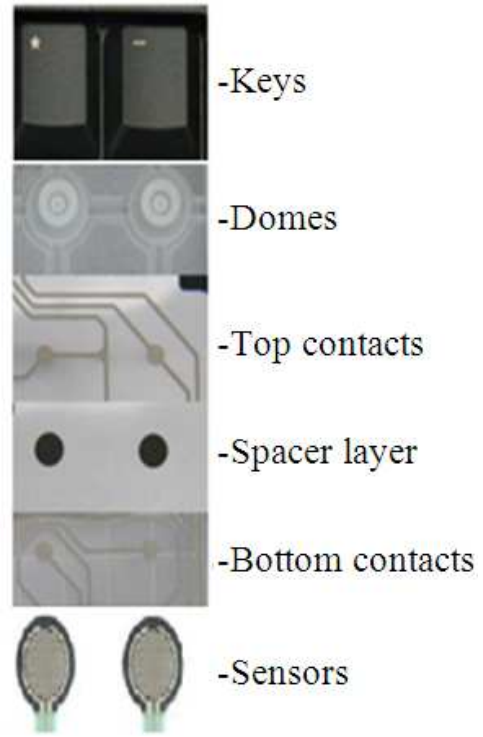

Fig 4. Keyboard layout with sensors placed

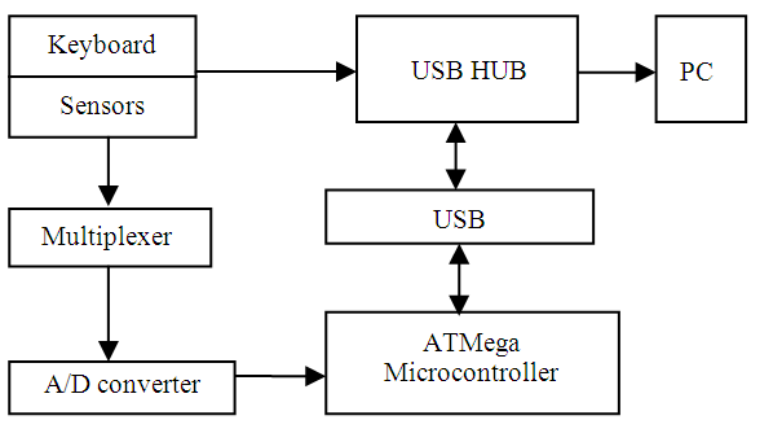

Fig. 5. System configuration of dual keyboard

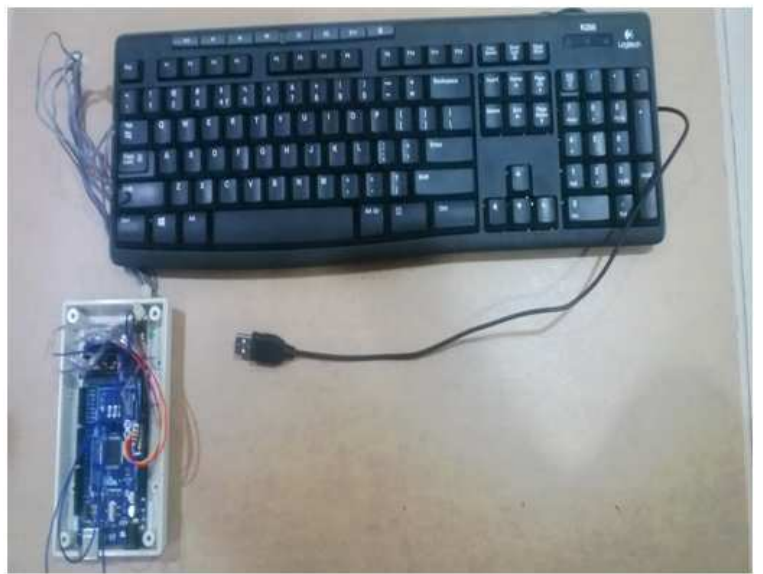

Fig. 6. Prototype of the dual keyboard

The Sensors are arranged in a row and column manner. The positive VCC is supplied to the one end of the sensor circuit using the ATMega development board. The other end of the sensor is connected to $10 \mathrm{k} \Omega$ fixed resistor. The resistor is then connected to ground. Current flows from the ATMega board, to the sensor. The output is taken across fixed resistor. When the pressure is applied to the sensor, the voltage across the $10 \mathrm{k}$ resistor varies according to the pressure applied. The varied analog voltage is converted in to digital using A/D converter. The sensor signal is then processed using Atmega microcontroller. By using multiplexer maximum number of sensors can be connected to the microcontroller. The output is sent to the PC via USB. The prototype of the Dual Keyboard is shown below in Fig. 6. In Fig. 7 the conceptual circuit is given.

\subsection{Working and Testing of Dual Keyboard}

Figure 8-12 respectively shows examples of keystrokes. The User has pressed the keys 'a', 'e', 'i', 'o', ' $u$ '. In the graph the upper wave represents the "Key Down" and the lower wave indicates the "Key Up". The user has repeatedly pressed each letter, which can be seen in the fluctuations of the graph. If the user presses harder the upper panel is more or it is short.

\subsection{Application-to Mitigate Denial of Service}

In this section, the Dual Keyboard while working as a pressure sensitive keyboard is used to mitigate denial of service is discussed. 
Ragavi, V. and G. Geetha / Journal of Computer Science 9 (11): 1566-1574, 2013

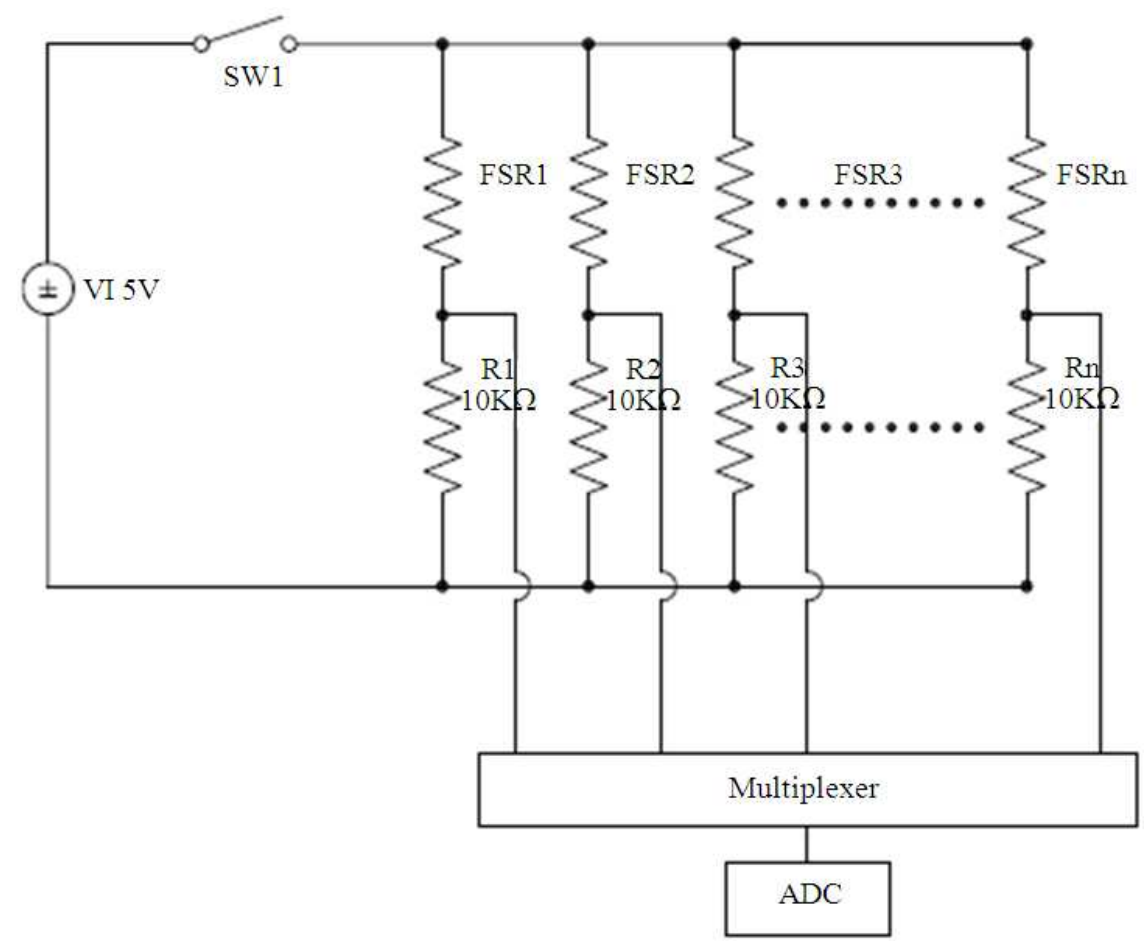

Fig. 7. Circuit for dual keyboard

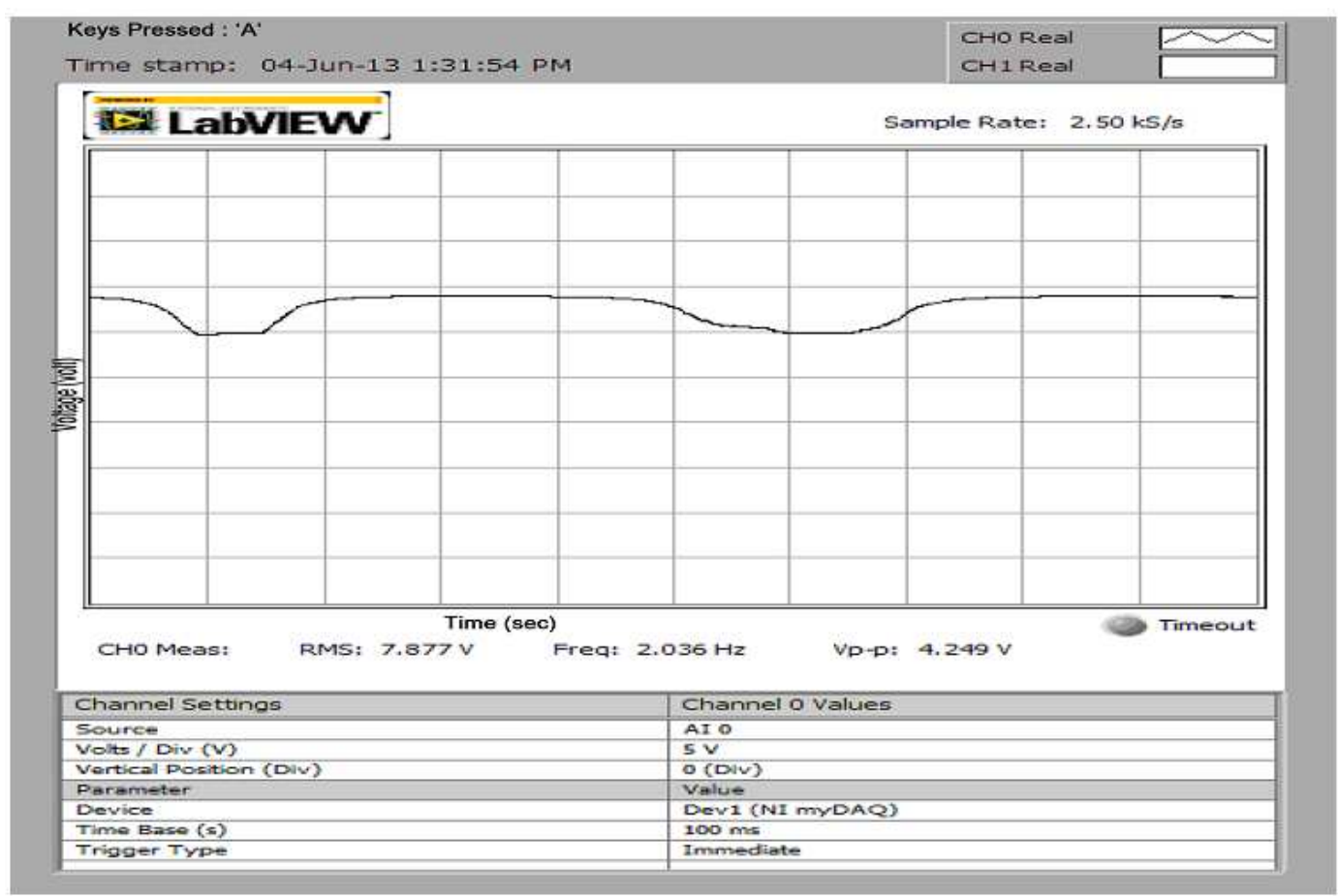

Fig. 8. Graph showing voltage fluctuation when the key ' $A$ ' is pressed 
Ragavi, V. and G. Geetha / Journal of Computer Science 9 (11): 1566-1574, 2013

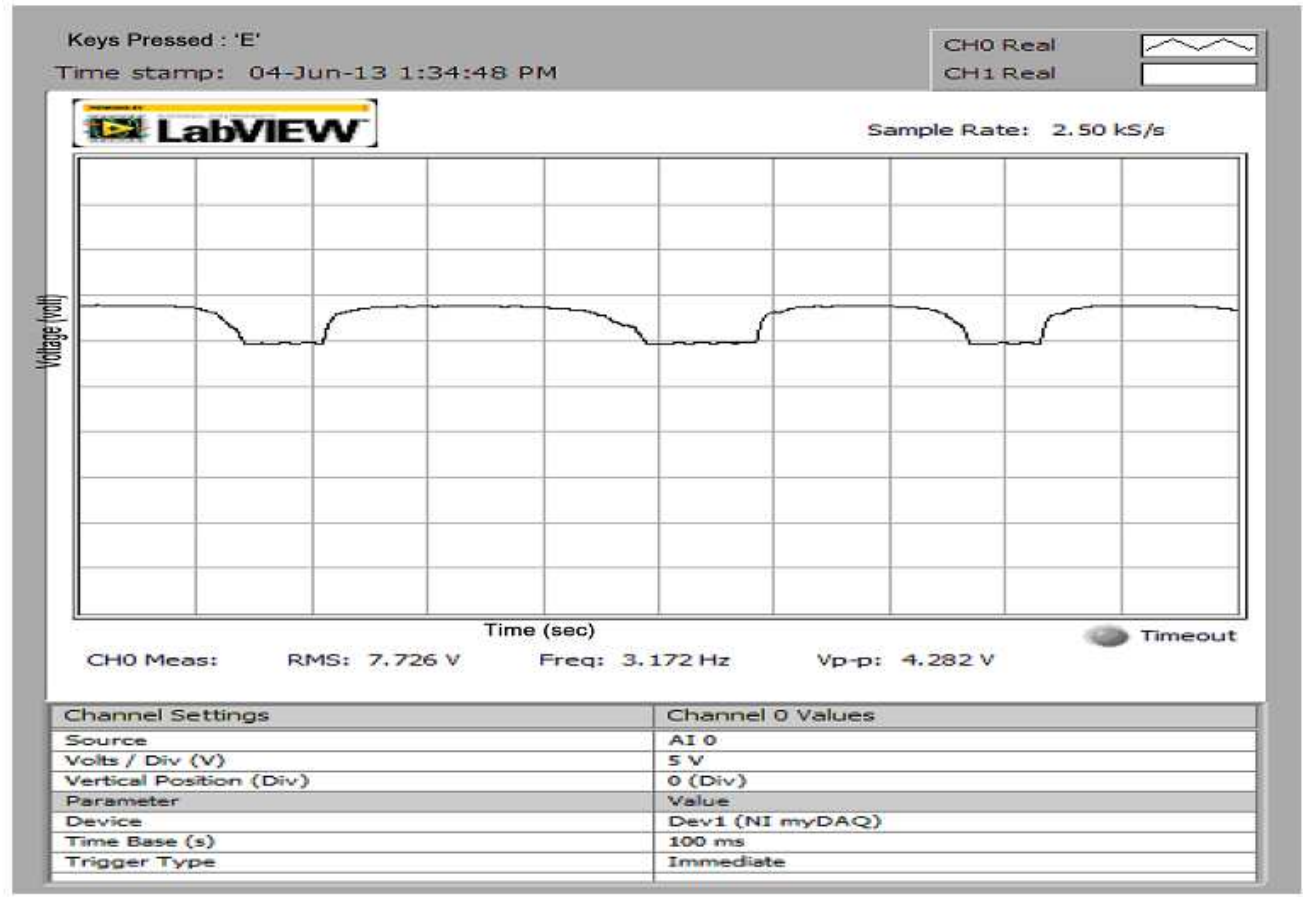

Fig. 9. Graph showing voltage fluctuation when the Key ' $E$ ' is pressed

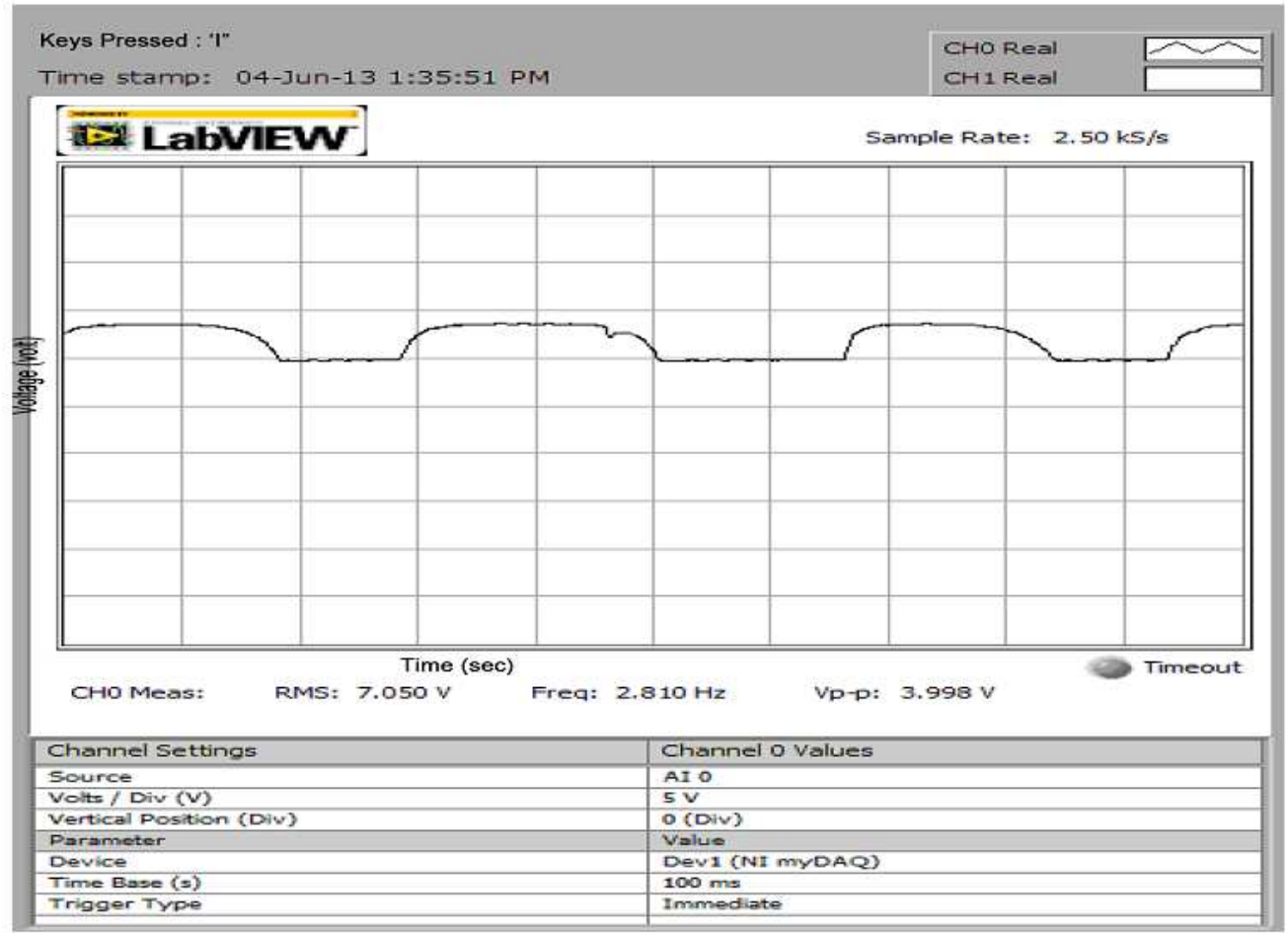

Fig. 10. Graph showing voltage fluctuation when the Key 'I' is pressed 


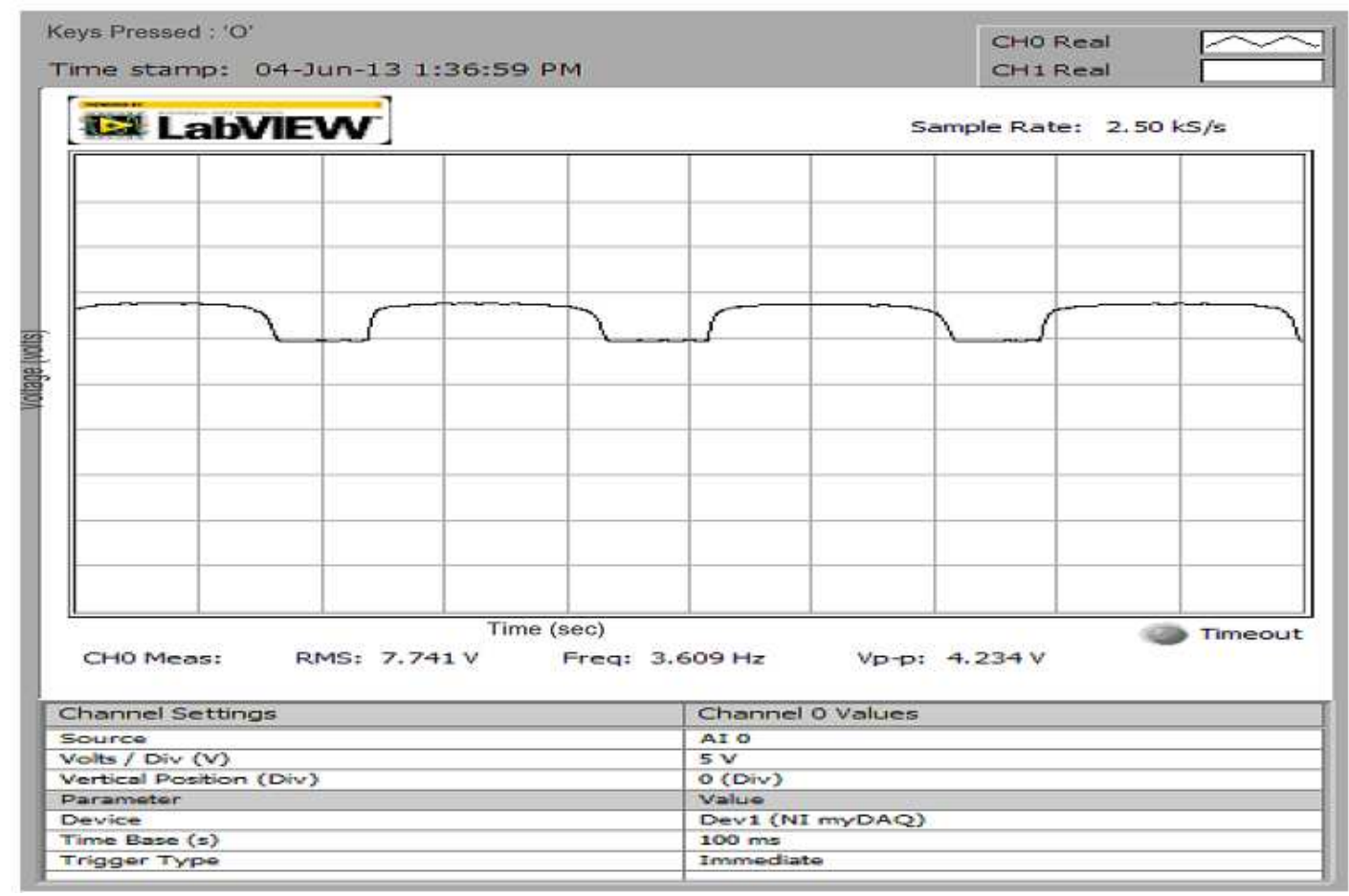

Fig. 11. Graph showing voltage fluctuation when the Key ' $\mathrm{O}$ ' is pressed

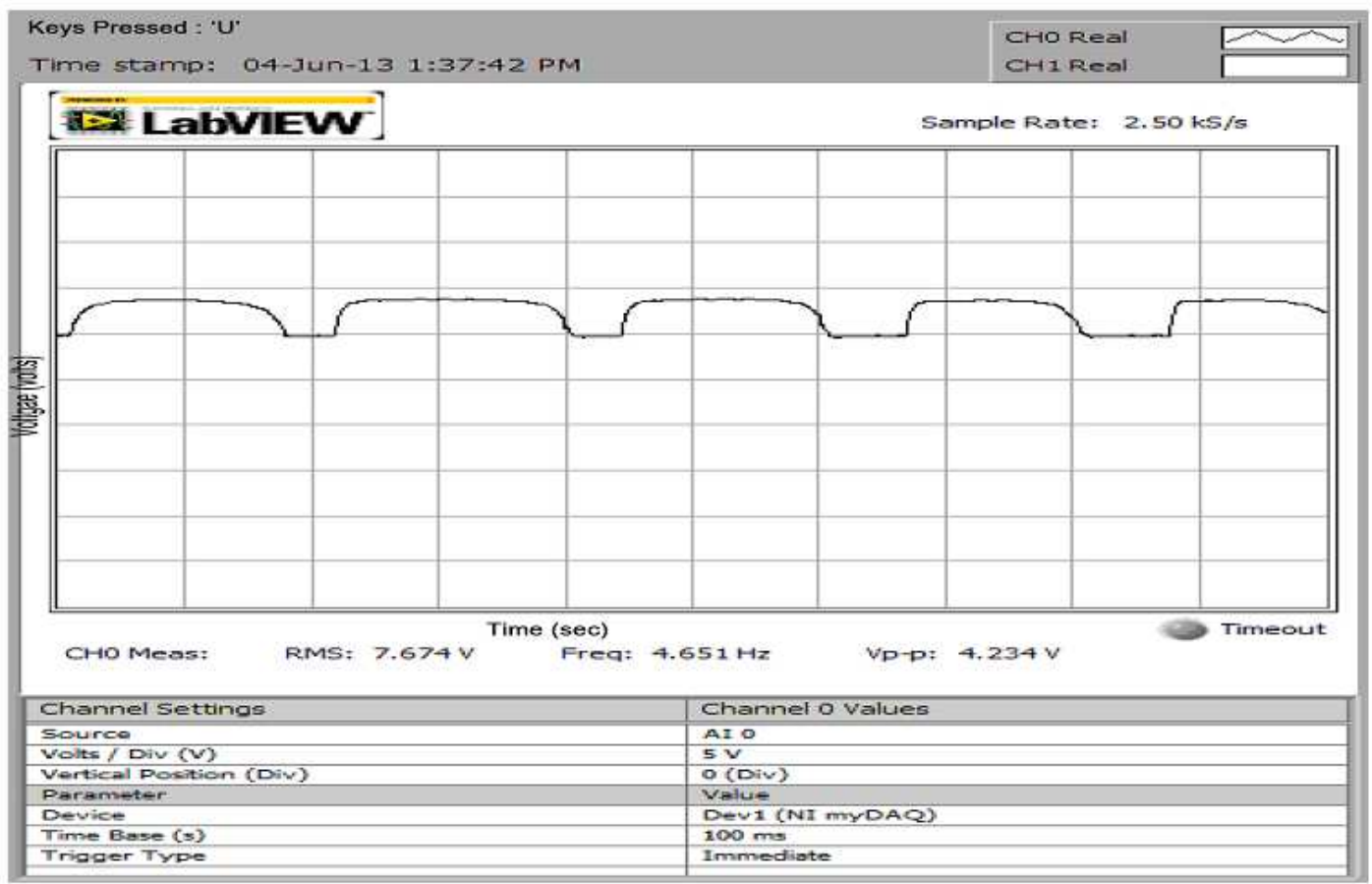

Fig. 12. Graph showing voltage fluctuation when the Key ' $U$ ' is pressed 


\subsection{To Mitigate DoS}

This innovative input device can be used to mitigate Denial of Service (DoS). The main goal of DoS attack on a network is to take out a large website by flooding it with useless traffic.

The aim is to stop the flooding by differentiating between man and bot. Techniques that try to detect such an attack and throttle down malicious traffic prevail today. CAPTCHA is one of the standard technologies used, which requires human-cognitive-processing abilities. Usability and robustness are two fundamental issues with CAPTCHA and they are often interconnected with each other. But, these are compromised by various factors. Usability of CAPTCHA has turned out to be of more consideration. CAPTCHA does not only have usability issues, it is also time consuming. About 200 million CAPTCHAs are solved by humans around the world every day. In each case, roughly ten seconds of human time are being spent. Individually, that's not a lot of time, but in aggregate these little puzzles consume more than 150,000 hours of work each day. What if one could make positive use of this human effort? It is discussed by various authors.

Section 4.2 will explain the process, how Dual Keyboard is used to distinguish man and bot.

\subsection{Process}

The User downloads any form (email registry, online application form) from the Application Server. As the User fills in the form with the Dual Keyboard, the pressure information is also embedded with the form data. The form is submitted. The submitted form is then evaluated to see whether pressure data exists. If pressure data exists, the form is accepted by the Application Server. As this form is filled by Human (it has manual entry). If pressure data does not exists then the form is discarded. As this form is filled by bot (automatic program), it does not have any manual entry. This concept is discussed by Ragavi and Geetha (2011; 2012a; 2012b).

\section{CONCLUSION}

As spammers advance, the puzzles get progressively harder and more burdens are placed on humans. What is required is a new test that does measures user heuristics without requiring additional user input. This innovative input device-Dual Keyboard, which is presented above is a solution to it.

The users will enjoy using dual keyboard than using the monotonous Computer Keyboard. Dual Keyboard can be inexpensively mass produced. The keyboard has other applications like Expressive Typing, Authentication, Gaming and many more.

The concept of pressure sensitivity can be extended to mouse, touch screen.

\section{REFERENCES}

Bergadano, F., D. Gunetti and C. Picardi, 2002. User authentication through keystroke dynamics. ACM Trans. Inform. Syst. Security, 5: 367-397. DOI: $10.1145 / 581271.581272$

Buxton, B., 2007. Multi-touch systems that I have known and loved. Microsoft Research.

Dietz, P.H., B. Eidelson, J. Westhues and S. Bathiche, 2009. A practical pressure sensitive computer keyboard. Proceedings of the 22nd annual ACM symposium on User Interface Software and Technology, Oct. 04-07, ACM Press, New York, USA., pp: 55-58. DOI: 10.1145/1622176.1622187

Henderson, N., N. White, R. Veldhiuis and P. Hartel, 2002. Sensing pressure for authentication. Proceedings of the 3rd IEEE Benelux Signal Processing Symposium, Mar. 21-22, Leuven, Belgium, pp: 1-4.

Henderson, N.J. and P.H. Hartel, 2000. 'Pressure sequence'-a novel method of protecting smart cards. Int. Federat. Inform. Process., 58: 241-256. DOI: 10.1007/978-0-387-35528-3_14

Hidetoshi, N. and M. Kurihara, 2007. Sensing pressure for authentication system using keystroke dynamics. World Acad. Sci. Eng. Technol., 1: 578581.

Loy, C.C., C.P. Lim and W.K. Lai, 2005. The development of a pressure-based typing biometrics user authentication system. Adv. Inform. Res. Group.

Ragavi, V. and G. Geetha, 2011. Usability issues of image based CAPTCHA. Proceedings of the National Conference on Network and Distributed Systems Security, Jan. 20-21, pp: 63-67.

Ragavi, V. and G. Geetha, 2012a. Mitigating DoS using sensing keys. Proceedings of the International Conference on Computing Sciences, Sep. 14-15, IEEE Xplore Press, Phagwara, pp: 312-315. DOI: 10.1109/ICCS.2012.46

Ragavi, V. and G. Geetha, 2012b. Usability issues of video based CAPTCHA. Proceedings of the National Conference on Emerging Trends in Computer Application, Feb. 24, pp: 127-131. 
Ramos, G. and R. Balakrishnan, 2005. Zliding: Fluid zooming and sliding for high precision parameter manipulation. Proceedings of the 18th Annual ACM Symposium on User Interface Software and Technology, Oct. 23-27, ACM Press, New York, USA., pp: 143-152. DOI: 10.1145/1095034.1095059

Ramos, G. and R. Balakrishnan, 2007. Pressure marks. Proceeding of the SIGCHI Conference on Human Factors in Computing Systems, Apr. 30-May 03, ACM Press, New York, USA., pp: 1375-1384. DOI: 10.1145/1240624.1240834
Ramos, G., M. Boulos and R. Balakrishnan, 2004. Pressure widgets. Proceeding of the SIGCHI Conference on Human Factors in Computing Systems, Apr. 24-29, ACM Press, New York, USA., pp: 487-494. DOI: 10.1145/985692.985754

Zagler, W.L., C. Beck and G. Seisenbacher, 2003. FASTY-faster and easier text generation for disabled people. Vienna University of Technology. 